\title{
Getting under your skin
}

utting aside the hype about a baldness cure, two recent works reporting epithelial stem cell isolation, one by Rebecca Morris et al. (1) and the second by Tudorita Tumbar et al. (2), provide more than just a skin-deep promise of future medical marvels. They may aid in revealing molecular mechanisms underlying several phenomena, including cellular differentiation, cancer development, and immune responses.

Recently, interest has grown for using adult stem cells instead of embryonic stem cells. The question, however, is whether adult stem cells are multipotent (able to divide into a select number of cell types) or pluripotent like embryonic stem cells (able to give rise to any cell type). While some data have suggested that adult stem cells can become pluripotent, these results have been difficult to replicate.

"Most of the evidence points to adult stem cells being somewhat lineage specific," George Cotsarelis, of the University of Pennsylvania and last author of Morris et al. (1), told the JCI. More practically, he added that "if you're looking for something to regenerate epidermal tissue, you may as well work with epidermal stem cells, though an intriguing question for us is [whether] these stem cells can become other cell types."

Given the approximately $95 \%$ purity in their stem cell assay, these cells may indeed be a

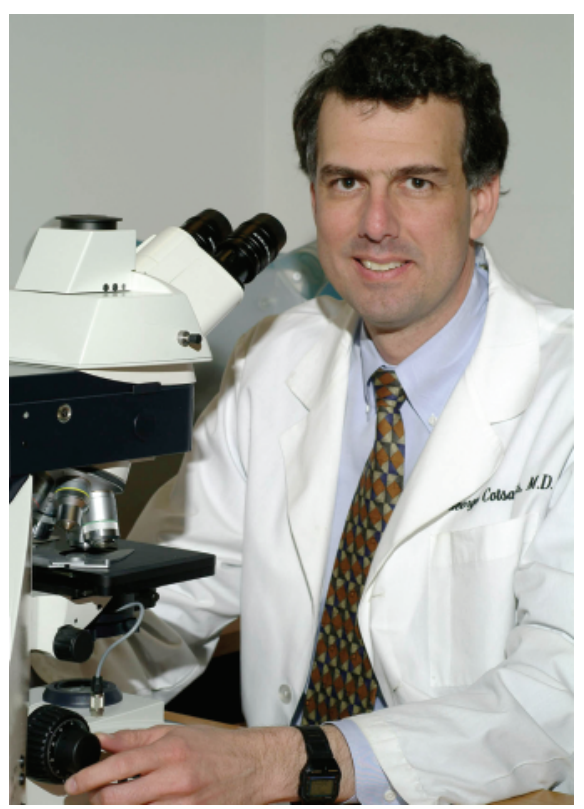

George Cotsarelis is looking for the means to isolate human epidermal stem cells. good source for addressing concerns from previous studies relating to contamination.

Much of the focus on the two reports, however, has been on their potential to cure baldness. Morris, of Columbia University, provided the JCI with some perspective.

"For years I have been trying to see if we could get hair follicle development from harvested skin cells from mice. This is the first time we ever have." She feels the greater purity of the stem cells here likely increased the chances for getting follicular growth.

"I wouldn't make the case that this immediately translates into a cure for baldness," she stated. "Maybe by identifying hair follicle stem cells and understanding how they interact with the cells and matrix proteins around them, we may be able to recreate hair."

Morris' main focus has been on the potential role epithelial stem cells play in skin cancer. Evidence indicates skin stem cells, rather than more differentiated cells, are the main source of skin cancer.

Morris and her colleagues believe the reason behind this is that "the stem cells and early progenitor cells are the only cells that have enough proliferative potential to divide enough times to form a tumor. The more-differentiated cells can divide, but ultimately get sloughed off before they can form tumors."

Morris feels that "understanding how the clonogenic cells are regulated will be important for understanding skin cancer."

Morris' group previously defined several mouse quantitative trait loci (QTL) related to keratinocyte stem cell proliferative potential. Their current work indicates that some of these QTL co-localize to skin cancer-linked regions. Further, one of the genes that were differentially expressed in their epithelial stem cells may produce a protein that interacts with the product of their gene of interest.

Likewise, focusing on other genes with altered expression in skin stem cells could enable the identification of other important molecular mechanisms.

Cotsarelis, commenting on the similarities and differences in the gene expression patterns seen in the microarray studies done in the two reports, noted that "what was striking were the similarities. Close to $40 \%$ of the genes were similar.”

That several histocompatibility genes were down-regulated in the stem cells is particularly intriguing to the authors.

Cotsarelis told the JCI, "This is really fasci-

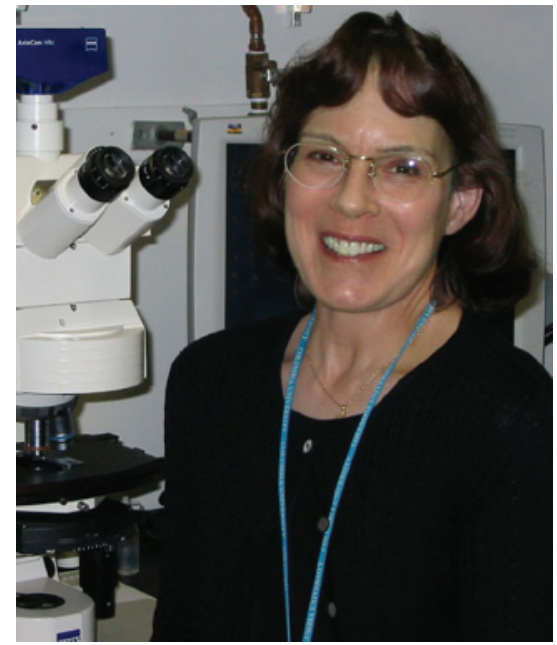

Rebecca Morris is pursuing genetic mechanisms relating epithelial stem cells to skin cancer.

nating because there are data around that suggests hair follicles are immune-privileged sites and when that immune privilege breaks down, it can cause certain types of hair loss.” Such may be the case for alopecia areata, an inflammatory disease, characterized by bald patches and hair follicle infiltration by $\mathrm{T}$ cells. Further analysis may provide the means to map the genetic components of hair follicle immune privilege and to understand how things go wrong.

For the future, Morris is particularly interested in utilizing skin stem cells to determine the lineage of genes that go on and off as epidermal cells differentiate. "Hopefully," she says, "we can do as well as the neurobiologists in determining the sequential developmental pathway in our stem cell system."

Cotsarelis agrees, adding that defining genes to isolate human epithelial stem cells is also a main focus. "We know the [human stem] cells are there; it is a matter of finding cell surface proteins. We have about 100 differentially expressed genes [from the mouse cells], so it is just a matter of screening through them. Others have shown that the genetics of the two systems [mice and human] are very similar; so the data here should be very useful."

\section{Laurie Goodman}

1. Morris, R.J., et al. 2004. Capturing and profiling adult hair follicle stem cells. Nat. Biotech. doi: $10.1038 / \mathrm{nbt} 950$

2. Tumbar, T., et al. 2004. Defining the epithelial stem cell niche in skin. Science. 303:359-363. doi: 10.1126/science.1092436. 